\title{
Detection of Defects in Automotive Metal Components Through Computer Vision
}

\author{
*Mário Campos, Teresa Martins**, Manuel Ferreira*, Cristina Santos* \\ University of Minho - Industrial Electronics Department - Guimarães, Portugal. \\ *Department of Industrial Electronics \\ University of Minho \\ Campus de Azurem - Guimarães, Portugal \\ Telf: +351253510190; fax: +351253510189; email: mario_campos@portugalmail.pt, \\ mjf@dei.uminho.pt, cristina@dei.uminho.pt \\ **Enermeter, Sistemas de Medição, SA \\ Praceta das Agras, 26 \\ Celeiros - Braga, Portugal \\ Telf: +351253287237; fax: +351253287238; email: tmartins@enermeter.pt
}

\begin{abstract}
This article introduces the progresses made on the development of an automatic visual inspection for automotive metal components. These components may present three types of defects, which are: central hole closed or partially closed; absence of thread on the bolts; burr on the weld connections. For each type of defect different image processing and analysis algorithms were tested and a specific setup (type and pose of the cameras and illumination) was developed. The results suggest a good efficiency of the vision system and its potential full spreading industrialization.
\end{abstract}

\section{INTRODUCTION}

Nowadays the quality control issues assume an important role in the manufactory of products and goods. With more and more demanding markets, companies are forced to invest on more efficient inspection systems, able to detect even the minor flaws during the production cycle. Computer vision techniques present a major key in this process of defects detection [1,2,3].

This article introduces the progresses made on the development of an automatic visual inspection for automotive metal components based on computer vision. Figure 1 shows two views of the metal pieces to be inspected. These components may present three types of defects, which are: central circular mark closed - hole (Figure 2), absence of thread on the bolts (Figure 3) and burr on the weld connections (Figure 4).
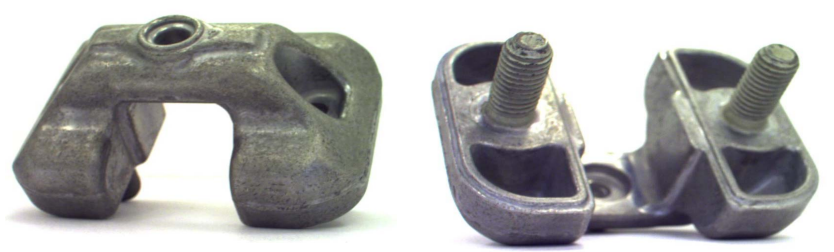

Fig. 1. Different views of the metal component to be analyzed.
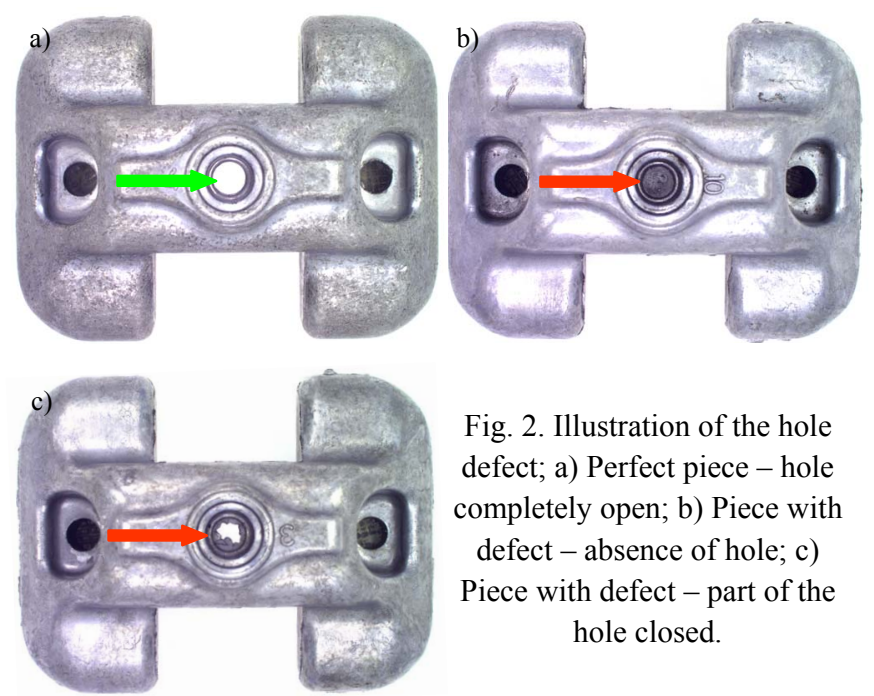

Fig. 2. Illustration of the hole defect; a) Perfect piece - hole completely open; b) Piece with defect - absence of hole; c) Piece with defect - part of the hole closed.

a)

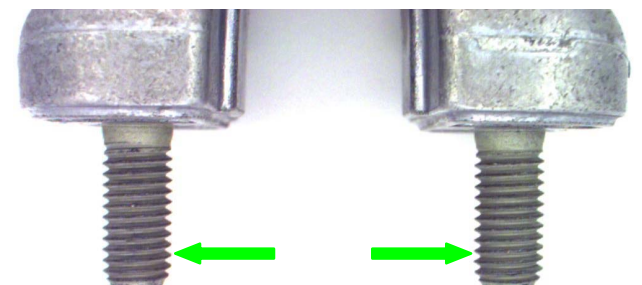

b)

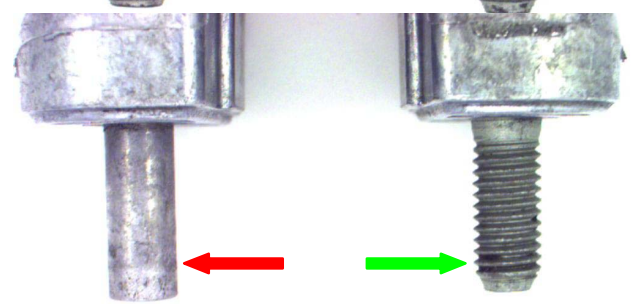

Fig. 3. Illustration of thread on the bolts; a) Perfect piece - presence of both threads; b) Piece with defect - absence of one thread. 
The defects are the result of problems during the injection process. Regarding the burr defect, present on the weld connections, it can be of the order of $10 \mu \mathrm{m}$.

For each type of defects different gray scale image analysis algorithms were tested. The results were obtained from a lab prototype that was developed simulating the industrial computer vision setup, designed specifically for this application.

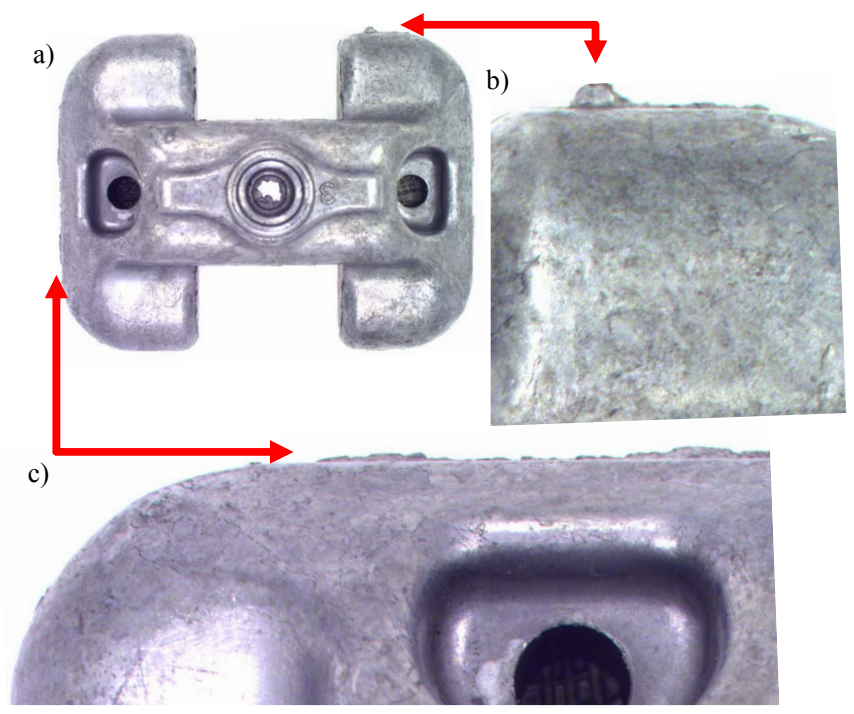

Fig. 4. Illustration of burr on the weld connections. a) front view image with burr. b) and c) burr detail images.

\section{METHODS}

\section{A. Close hole defect}

The hole is situated in the centre of the metal piece and, as can be observed in the front view of the metal component (figure 2), it is surrounded by a circular metal structure (circular mark). Figure 5 shows the block diagram of the two approaches developed to detect this defect. In both cases the crucial step is the detection of the hole position (or the circular mark) in the image. The hole is considered open if there are pixels inside the circular mark with the same gray level of the background performing a binarization with a threshold level $\mathrm{T}$. One of the approaches is based on the circular Hough transform whereas the other uses the contour boundingbox.

The circular Hough [4,5,6] transformation finds several circles on the image. From all the potential circles, the correct one must be determined. This is done using the centre of the circle and the circle diameter. Once acknowledged the circle, the pixels inside are examined in order to check if its colour is the same as the background.

The other approach consists in extracting the contours of the metal component and calculating the rectangle with minimal area which circumscribes the component boundingbox [7]. The centre of the rectangle should be the centre of the hole. Once recognized the centre of the component the pixels around it are analyzed in order to check if its gray level is the same as the background. This approach needs to establish previously the radius of the circle mark.
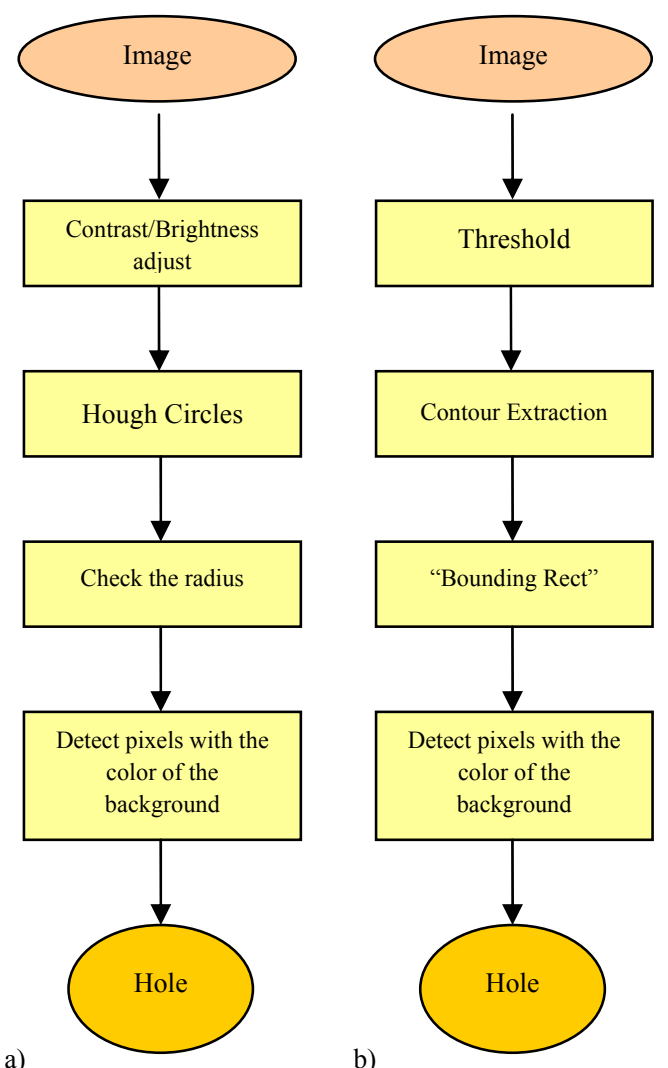

Fig. 5. Block diagrams of the two approaches used to detect the close hole defect. a) Circular Hough transform. b) BoundingBox.

\section{B. Absence of thread defect}

Both bolts should have a thread. The approach used to detect these threads consists on evaluating the continuity of the bolt edges. The algorithm analyses the periodic behaviour which is like a triangular signal - of the edges.

The diagram of figure 6 shows the procedure that was implemented to detect this defect. The first step consists in separating the bolts from the body of the component and then to process each one independently.

The diagram of figure $6 \mathrm{~b}$ describes the study of the periodic behavior of the edges. If the period of the edges occurs in a pre-defined number of blocks with a pre-defined size it means that the bolt has a thread with the correct specifications. Figure 7 and 8 show the two distinct situations: the presence of thread and the absence of thread. Each figure contains the binary bolt image with the region of interest (bolt) demarked. Also it is presented the values (in a chart) of a line image. A quasi perfect triangular signal can be observed for the bolt with the thread (figure $7 \mathrm{~b}$ ), which is not the case for the bolt without it (figure 8b). 


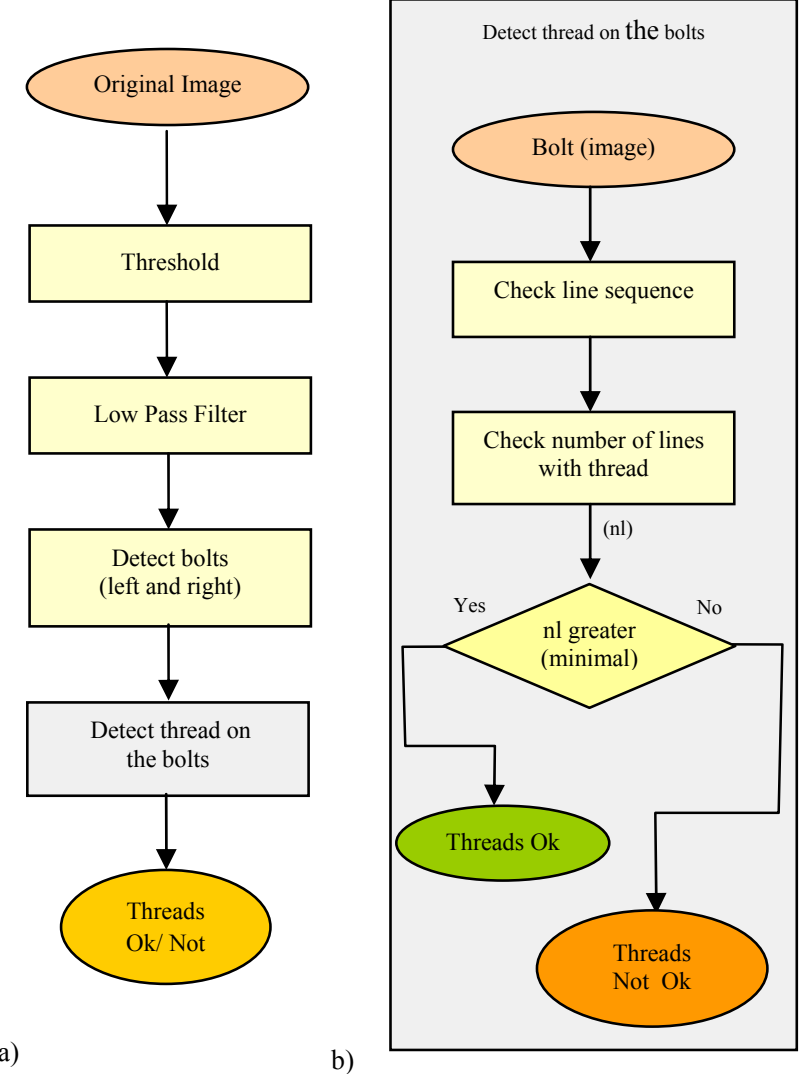

Fig. 6. Block diagrams of the approach used to detect the absence of the thread. a) Overall procedure. b) Algorithm for thread evaluation.

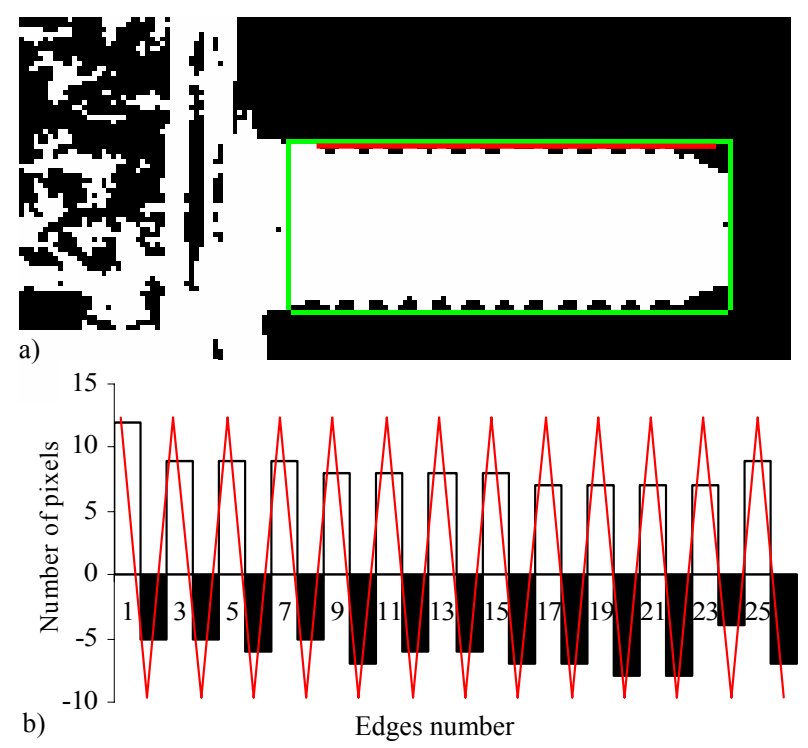

Fig. 7. Binary image of a bolt with the thread. a) Bolt region of interest for thread evaluation (green rectangle) and the mark of the line analyzed in b). b) Chart with the triangular behavior (red line) and the number of pixels of the white and black parts of the line image (bar chart). For visualization only the number of pixels of the black part was multiplied by -1 .

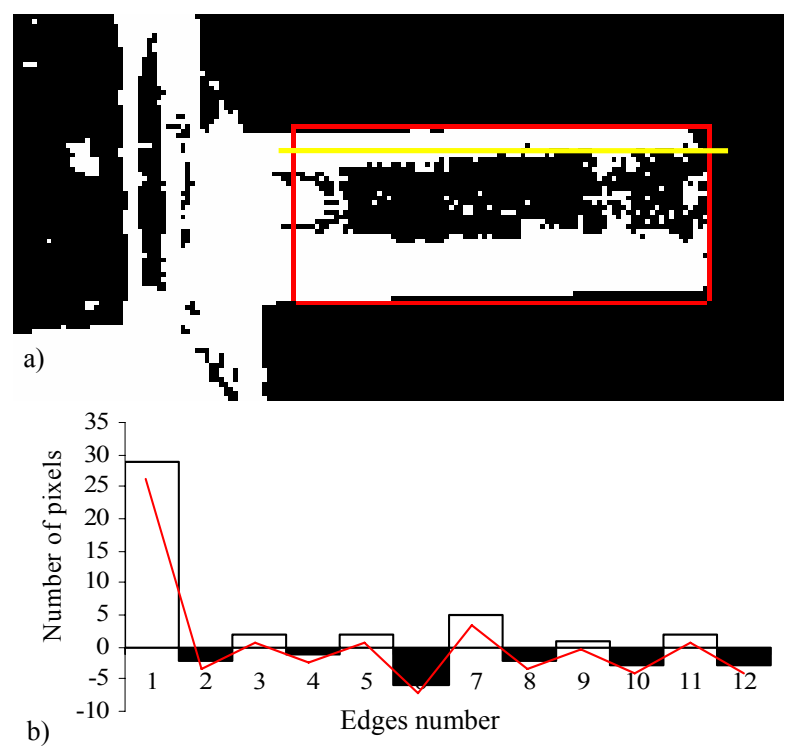

Fig. 8. Binary image of a bolt without the thread. a) Bolt region of interest for thread evaluation (red rectangle) and the mark of the analyzed in b). b) Chart with the non-triangular behavior (red line) and the number of pixels of the white and black parts of the line image (bar chart). For visualization only the number of pixels of the black part was multiplied by -1 .

\section{Burr defect}

To identify the burr, an analysis of the component contour was developed. First, the approach consists in the correction of the component pose to a known pose. This must be done because in an industrial environment the component can appear in a rotated position. By correcting the rotation angle the burr detection becomes simpler, because it is now possible to compare the contour with the predicted contour of the ideal (without defect) component. The angle is detected using minimal-area bounding rectangle of the contour. After obtaining the rotation angle, the image is rotated with the reverse value. Figure 9a shows the diagram of the image pose correction procedure. After the pose correction the contour is extracted and then is submitted to a check quadrant algorithm (figure 9b).

According to this algorithm the piece is divided in 10 sectors (figure 10). For each sector the displacement directions from a contour point to a consecutive one occur inside the same quadrant (quadrant 1: directions from $0^{\circ}$ to $90^{\circ}$; quadrant 2: directions from $90^{\circ}$ to $180^{\circ}$; quadrant 3: directions from $180^{\circ}$ to $270^{\circ}$; quadrant 4: directions from $270^{\circ}$ to $360^{\circ}$ ). As an example figure 11 a shows the sector 1 with the respective legal directions belonging to the quadrant 3 (green arrows); the red arrows represent illegal directions. In that way, when a burr is present there are shifts in the contour directions leading to detection of displacements on other quadrants (Figure 11b). In other words, if the quadrant obtained is not the predicted one for that sector this indicates the presence of burr. 


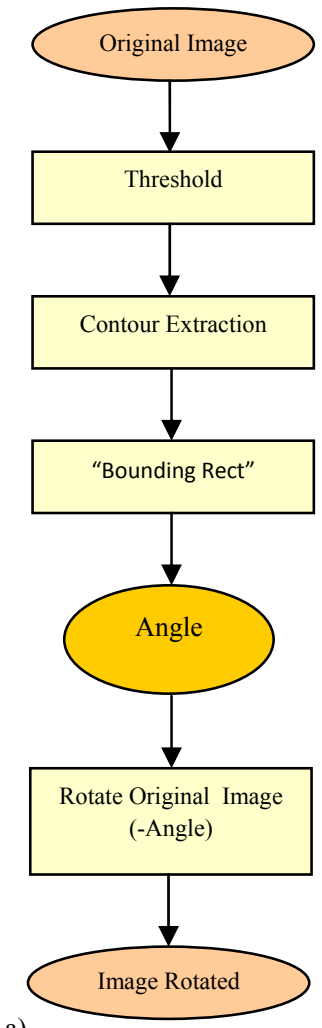

a)

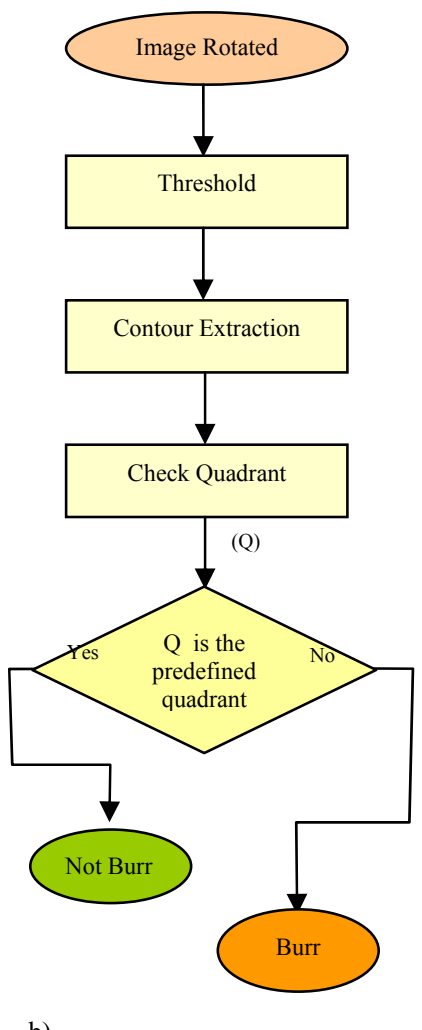

b)

Fig. 9. Block diagram of the burr analysis procedure. a) pose detection and image rotation procedures. b) Burr evaluation algorithm.

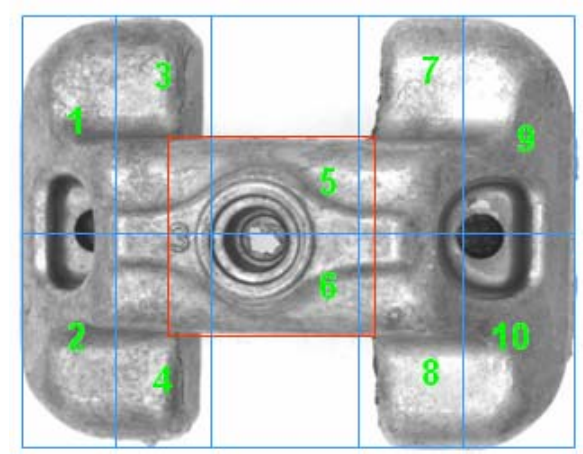

Fig. 10. Definition of sectors for burr analysis.

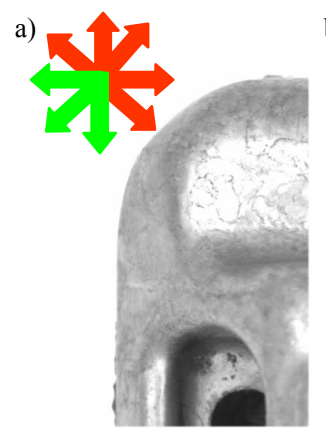

b)

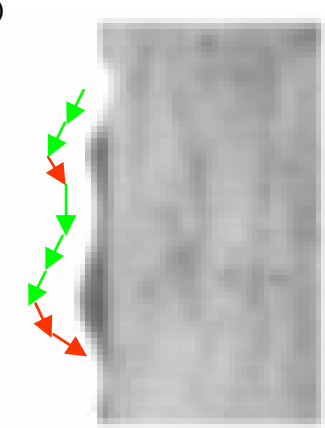

Fig. 11. Illustration of the check quadrant procedure. a) Legal (green) and illegal (red) directions for sector 1. b) Shift in directions in presence of a burr.

\section{RESULTS AND DisCUSSION}

In order to test the different algorithms and the acquisition system a laboratory prototype was constructed. The figure 12 shows the lab acquisition prototype and a representation of the future integration at the industrial environment.

The setup conditions are essential to get the best picture for image processing. So, the option for a suitable setup strongly influences the results, especially concerning the cameras pose, the lightning and the background colour. The latest affects the lightning distribution; consequently it is desired to use a white colour on the background. The lightning used is a fluorescent like light.

Due to the geometry of the metal component two acquisition systems were used. One is the camera for detection of the absent thread on the bolts, which is placed perpendicular to the bolts. For detection of the hole and the burrs, another camera is placed frontally to the top of the metal component.

The algorithms were implemented in $\mathrm{C}++$ and made use of the computer vision library - OpenCv[8]. The cameras are color firewire from Allied with CCD progressive and resolution of $3272 \times 2469$ pixels. The image was acquired in monochromatic mode.

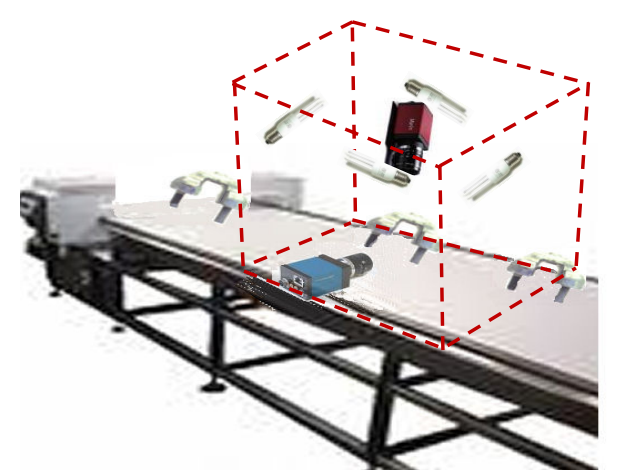

Fig. 12. Lab acquisition prototype with a possible representation of future integration at the industrial environment.

\section{A. Close hole defect}

After a several number of tests the results show that both methods - Hough, boundingbox - work well with different advantages for each one.

The figure 13 and 14 show the results obtained with the Hough transformation method and the boundingbox method applied to three distinct component images, respectively.

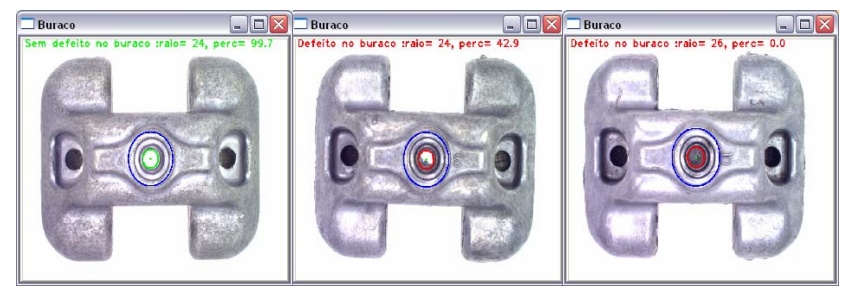

Fig. 13. Application of circular Hough transformation. 


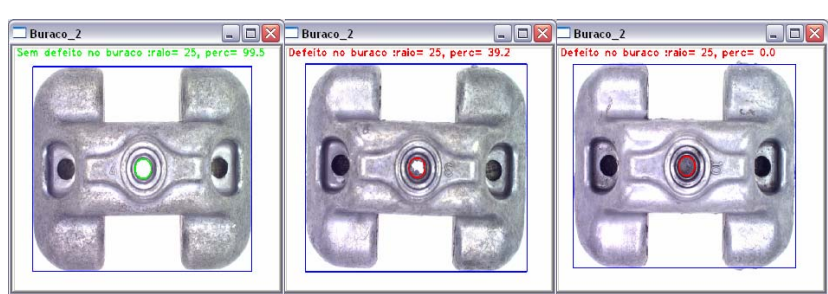

Fig. 14. Application of Boundingbox.

The blue circles in figure 13 are the ones detected by the Hough transform and the blue rectangles of figure 14 are the boundingboxes used for the detection of the centre. The left images of both figures correspond to the open hole detection without defect (green circle). The others (middle and right) show the detection with defect (red circle).

The Hough transformation takes more processing time but is more precise because does not need to establish a previous hole radius and is not affected by the presence of a significant burr. The main disadvantage is that some drift in the illumination, or changes in the metal properties of the component (for instance specular reflection) can cause a non detection of the circle or an erroneous detection.

The boundingbox approach does not calculate the hole radius, which means it has to be pre-defined and it is not flexible to some changes on the component. Also, the presence of a significant burr can affect the bounding box centre. To minimize this effect a morphologic filter applied to a higher level in a pyramid decomposition of the image can be used.

Taking into account these aspects, the boundingbox approach was the one selected to integrate the overall system. The hole is considered to be completely open if $96 \%$ of the pixels are of the same gray level of the background. This parameter and the binarization threshold $\mathrm{T}$ are dependent on the type of background, illumination conditions and for that they are two setup parameters of the system (Table I).

TABLE I

SETUP PARAMETERS

\begin{tabular}{|c|c|}
\hline \multicolumn{2}{|c|}{ Hole detection } \\
\hline Minimum radius value & 90 \\
\hline Maximum radius value & 120 \\
\hline Binarization threshold T & 222 \\
\hline \%Gray level background pixels & 96 \\
\hline Absent of thread \\
\hline Binariazation threshold & 244 \\
\hline Minimum pixels between thread & 4 \\
\hline Maximum pixels between thread & 17 \\
\hline Number of minimum thread & 10 \\
\hline Number of lines to analyse & 15 \\
\hline Burr detection \\
\hline Binarization threshold & 244 \\
\hline Depth of burr & 2 \\
\hline
\end{tabular}

\section{B. Absence of thread defect}

This method can separate the bolts from the piece body and find the existence or not of the threads on them. The figure 15 shows the results for defected and non defected pieces, identifying the bolt with defect (red box). For the correct ones the system also indicates the number of threads which is a functionality that can be useful in future inspections like verifying the type of thread.

To increase the robustness of the solution a total of 15 lines in each side of the bolt is analysed. A set of setup parameters must be specified: binarization threshold (in the Detect bolts procedure of figure 6); number of lines to analyse in each bolt; number of edges in each line; number of pixels between edges in each line (Table I).

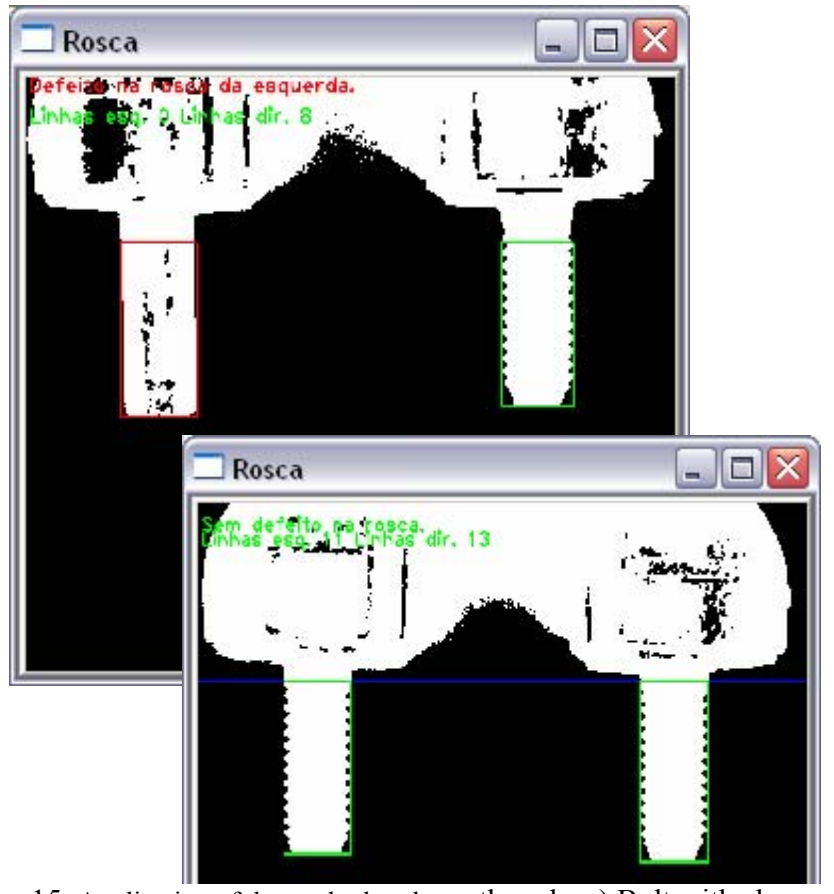

Fig. 15. Application of the method to detect threads. a) Bolt with absence of thread, b) Bolt without defect.

\section{Burr defect}

The burr is detected according to the not concur to the predefined quadrant, as depicted earlier in the methods section. The figure 16 shows the result obtained in the presence of a burr.

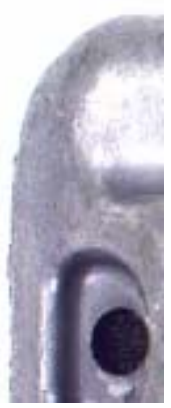

b)
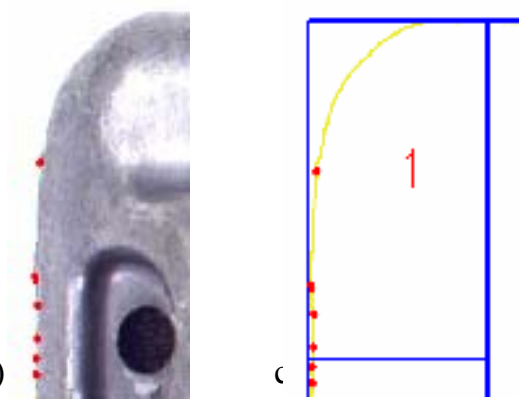

Fig. 16. Burr detection, a) piece sector 1 with burr b) burr detection result over the sector $\mathrm{c}$ ) burr detection result over the contour. 
In order to allow the separation of different burr intensities a setup parameter must be specified. This parameter consists on the number of pixels in directions with opposite quadrants. It is dependent of the image resolution and contrast and for burr detection with a higher accuracy must have a lower value of 1 or 2 pixel (Table I).

This procedure detects only the end of a burr, once it is backing to its original position (Figure 11b). However it shows a high efficiency to detect the distinct burr defects tested. New developments are under taking in order to detect the beginning of the burr. In that way the size of the burr can also be used also as a quality parameter.

\section{CONCLUSIONS}

A computer vision system was developed to detect defects in automotive metal components. Due to the type of material a dedicated industrial setup was specified and a lab prototype was developed for testing performance.

For the different type of defects - close hole; absence of threads; presence of burr - specific image processing and analysis algorithms were developed. For each defect a set of setup parameters were identified to allow a higher flexibility of the system. The tests performed on the lab prototype indicate that full industrialization of this approach is a promising perspective.

\section{REFERENCES}

[1] Rafael C. Gonzalez, Richard E. Woods, "Digital Image Processing”, Prentice Hall, 1992.

[2] John C. Russ, "The Image Processing Handbook", 2dh edition, CRC Press, 1995.

[3] Paul Suetens, Pascal Fua, Andrew J. Hanson "Computational Strategies for Object Recognition", ACM Computing Surveys, Vol 24, No 1, March 1992.

[4] H.K. Yuen, J. Princen, J. Illingworth and J. Kittler. Comparative study of Hough Transform methods for circle finding. Image and Vision Computing, Elsevier Science, Volume 8, issue 1, Feb 1990, 71-77.

[5] T. D'Orazio, N. Ancona, G. Cicireli, M. Nitti, A Ball Detection Algorithm for Real Soccer Image Sequences, Proceedings. 16th International Conference on Pattern Recognition, Volume 1, 2002, pp. $210-213$.

[6] Jung Rack Kim, Jan-Peter Muller, Jeremy G Morley, "Quantitative Assessment of Automated Crater Detection on Mars", XXth ISPRS Congress, 12-23 July 2004 Istanbul, Turkey.

[7] Dana H. Ballard, Christopher M. Brown, Computer vision, New Jersey: Prentice Hall, 1982.

[8] OpenCV, "Open Source Computer Vision Library", in http://netaro.ddo.jp/techinfo/OpenCV/opencv094-man/index.htm, (03-09-2007) 